\title{
Life table invasion models: spatial progression and species-specific partitioning
}

\author{
Zihua Zhao, ${ }^{1,10}$ Cang Hui,${ }^{2,3}$ Richard E. Plant, ${ }^{4}$ Min Su, ${ }^{5}$ Tim Carpenter,${ }^{6}$ Nikos Papadopoulos, ${ }^{7}$ Zhihong Li, ${ }^{1}$ \\ AND JAMES R. CAREY 8,9 \\ ${ }^{1}$ Department of Entomology, College of Plant Protection, China Agricultural University, Beijing 100193 China \\ ${ }^{2}$ Centre for Invasion Biology, Department of Mathematical Sciences, Stellenbosch University, Matieland 7602 South Africa \\ ${ }^{3}$ Mathematical and Physical Biosciences, African Institute for Mathematical Sciences, Muizenberg 7945 South Africa \\ ${ }^{4}$ Departments of Plant Sciences and Biological and Agricultural Engineering, University of California, Davis, Davis, California 95616 \\ USA \\ ${ }^{5}$ School of Mathematics, Hefei University of Technology, Hefei 230009 China \\ ${ }^{6}$ School of Veterinary Medicine, University of California, Davis, Davis, California 95616 USA \\ ${ }^{7}$ Laboratory of Entomology and Agricultural Zoology, School of Agricultural Sciences, University of Thessaly, Thessaly 38446 Greece \\ ${ }^{8}$ Department of Entomology, University of California, Davis, California 95616 USA \\ ${ }^{9}$ Center for the Economic and Demography of Aging, University of California, Berkeley, California 94720 USA
}

Citation: Zhao, Z., C. Hui, R. E. Plant, M. Su, T. Carpenter, N. Papadopoulos, Z. Li, and J. R. Carey. 2019. Life table invasion models: spatial progression and species-specific partitioning. Ecology 100(5):e02682. 10.1002/ecy.2682

Abstract. Biological invasions are increasingly being considered important spatial processes that drive global changes, threatening biodiversity, regional economies, and ecosystem functions. A unifying conceptual model of the invasion dynamics could serve as a useful tool for comparison and classification of invasion processes involving different species across large geographic ranges. By dividing these geographic ranges that are subject to invasions into discrete spatial units, we here conceptualize the invasion process as the transition from pristine to invaded spatial units. We use California cities as the spatial units and a long-term database of invasive tropical tephritids to characterize the invasion patterns. A new life-table method based on insect demography, including the progression model of invasion stage transition and the species-specific partitioning model of multispecies invasions, was developed to analyze the invasion patterns. The progression model allows us to estimate the probability and rate of transition for individual cities from pristine to infested stages and subsequently differentiate the first year of detection from detection recurrences. Importantly, we show that the interval of invasive tephritid recurrence in a city declines with increasing invasion stages of the city. The species-specific partitioning model revealed profound differences in invasion outcome depending on which tephritid species was first detected (and then locally eradicated) in the early stage of invasion. Taken together, we discuss how these two life-table invasion models can cast new light on existing invasion concepts; in particular, on formulating invasion dynamics as the state transition of cities and partitioning species-specific roles during multispecies invasions. These models provide a new set of tools for predicting the spatiotemporal progression of invasion and providing early warnings of recurrent invasions for efficient management.

Key words: demography; detection; eradication; invasion ecology; life table; tephritid.

\section{INTRODUCTION}

Biological invasions into wholly new areas are the consequence of a far-reaching but underappreciated component of global environmental change: the humancaused breakdown of biogeographic barriers to species dispersal (Dantonio and Vitousek 1992, Kriticos and Leriche 2010). To realistically capture the post-introduction invasion dynamics, many quantitative models have been developed, utilizing a range of mathematical and statistical

Manuscript received 26 January 2019; accepted 4 February 2019. Corresponding Editor: Gordon A. Fox.

${ }^{10}$ E-mail: zhzhao@cau.edu.cn tools (e.g., dispersal kernel models and species distribution models; Kearney and Porter 2009, Vicente et al. 2016, Cassey et al. 2018) but mostly only focusing on the spread of one focal invasive species over heterogeneous habitats (Hui and Richardson 2017). Management prioritization for post-introduction invasion has normally been based on characters of both invasive species and invaded sites (McGeoch et al. 2016), although a site-based dynamic framework of invasion remains lacking. Moreover, multispecies invasion could trigger site-specific invasion meltdown (Simberloff 2006), while site-based assessment facing multispecies invasions is still understudied.

All biological invasions share three mutually dependent, inextricably linked elements: spatiality, 
temporality, and presence in ecology (Roques et al. 2016). The first refers to the fact that biological invasions occur within and between confined regions, the second to the time component involved in colonizing one region and spreading over others, and the third to whether the invader has been detected within a given region (Epanchin-Niell and Liebhold 2015, Thomas et al. 2017). The third component can be further subdivided into whether presence refers to the first detection or, more importantly, recurrent ones (often after successful past eradications). Collectively, these three elements allow invasion dynamics to be re-conceptualized as an actuarial process in which sites (or regions) are individual entities initially in the state of absence but subject to the risk of transitioning into the new state of (first-detection) presence and beyond (recurrent stages; Manrakhan et al. 2015, Bertelsmeier and Keller 2018). Considering sites under multispecies invasions could further complicate the potential states and stages of these sites if we were to differentiate the scenario of co-occurrence and the sequence of first and recurrent invasions from different invasive species.

It is widely known in demography (Preston et al. 2001, Land et al. 2005) and to a lesser extent in ecology (Koons et al. 2014) that the life table is one of the most adaptable models. Life table models have been applied not only to analyzing survival and mortality as it was originally conceived over four centuries ago (Graunt 1662), but also to examining a wide range of single decrement processes. These include nuptiality, migration, and incarceration in human demography (Preston et al. 2001, Lamb and Siegel 2004), and diapause, sexual maturation, and plant flowering in ecology (Carey and Roach 2019). Indeed, the full range of life table methods involves some of the most versatile, adaptable, scalable, intuitive, and generalizable models in all of science (Carey 2001). These models encompass not only the standard cohort life table, but also important extensions such as the health life table (HLT; Coutsoudis et al. 1999), which studies transitions between different health states, and the multiple decrement life table (MDLT), which studies different ways to exit life such as through accident or disease (Lloyd-Jones et al. 1999, Duan et al. 2014).

The HLT model has been applied to describing life history, interspecific relationships, and the evolution of insects (Chi 1988, Bellows et al. 1992), and we argue here that it can also be used to describe the spatial dynamics of invasion progression. As we will show later, the concept and method for modeling invasion progression can be derived from life table concepts similar to those used in human health models, such as active life expectancy, Grade-of-Membership models (Lamb and Siegel 2004), classification of health-related states (WHO 2001), and cancer staging (Amin et al. 2017). The state of healthy "membership" can be important in invasion ecology for similar reasons in the health sciences: it provides a scientific basis for classifying sites at different invasion stages based on detection frequency, thus bringing the HLT model to bear on the site-based invasion dynamics.

The MDLT method is normally used in actuarial science when individuals are subject to more than one risk of dying (i.e., multiple causes of death). The MDLT is concerned with three questions: (1) the distribution of death from different causes often acting simultaneously; (2) the probability of a newborn dying from a specified cause; and (3) changes in the mortality patterns if certain causes are eliminated (Carey 1989). The MDLT has been applied to the analysis of species-specific cause of death in insect pest populations from multiple natural enemies (Peterson et al. 2009). In particular, the species-specific partitioning in the MDLT method (Lamb and Siegel 2004, Land et al. 2005) explores competing sources of risk (Ergon et al. 2018) and can be brought to bear on multispecies invasions.

We here exemplify the HLT and MDLT models by investigating the invasion of multiple tephritidae species over counties and cities of California. There have been 17 alien tephritids reported in California, starting with the Mexican fruit fly (Anastrepha ludens) and the oriental fruit fly (Bactrocera dorsalis), first detected in 1954 and 1960, respectively (Papadopoulos et al. 2013). Both the invasion and management response of alien tephritids are intensive but idiosyncratic in California, including state-wide intensive monitoring programs $(>10,000$ traps for $>50 \mathrm{yr}$ ) with high precision of detection and species identification, and emergency eradication programs in cities with invasive tephritids detected. Both the impact of tephritid invasion to the agricultural sector and the running of the two programs have to date cost the state of California more than US\$10 billion (Carey et al. 2017) and, consequently, the spatiotemporal dynamics of tephritid invasion has received much attention among ecologists in recent decades (Zeng et al. 2019). We extend the life table models to framing the invasion dynamics of alien tephritids in California with spatial units (geographic sites) as individuals in the life table. In doing so, we aim to achieve three specific objectives: (1) demonstrating how biological invasions can be framed as actuarial processes by defining and circumscribing spatial units and dichotomizing their status as infested or not; (2) introducing the invasion progression and species-specific partitioning models; and (3) exploring policy implications of invasion processes and management from the life table models.

\section{Methods And Materials}

\section{Database description}

The study area encompasses the entire state of California, which has a variety of climates from hot desert to subarctic depending on locations (Appendix S1: Fig. S1). The host plants of alien tephritids, including avocado, citrus, mango, and varieties of pome and stone 
fruit, have a wide distribution in California. The trapping strategy of using both pheromone (Methyl eugenol) and protein baits for capturing tephritids was developed by the California Department of Food and Agriculture (CDFA) and has been widely used for monitoring alien tephritids since 1950 (Steiner 1952). The historical data set (1950-2017) was provided by the CDFA for this study. To supplement, we also retrieved the detection monitoring reports from the California Plant Pest and Disease Reports (CPPDR), Mediterranean fruit fly detections (1975-1994) from the database of the last author at the University of California and from the CDFA website for recent updates from 2012 to 2018 (data available at: https://www.cdfa.ca.gov/plant/pdep/ treatment/index.html). ${ }^{11}$ Each detection record includes species, time (year/month/date), city, number of individuals, and precise location. We defined a detection event as the capture of $\geq 1$ alien tephritid in a city in one particular year, and the detection-year (DY) of a city as the number of years with positive detections in the city from 1950 to a specific year of concern. Note, once detected in a city, an emergency eradication program (quarantine treatment) will immediately kick in to suppress the alien tephritids within the city to below the detection threshold within the same year of detection (CDFA 2018). For each city in California, we thus produced a 68 -yr time series of the annual presence/absence for each tephritid species from 1950 to 2017.

\section{Conceptual framework}

We considered cities in California as a cohort of pristine (pre-invaded) habitats subject to the invasion from alien tephritids. Given a city as the initial point of invasion, we can schematically consider a $3 \times 3$ grid of nine cities, centered on the introduction city (Fig. 1). The population of alien tephritids may then propagate from the introduction city into other ones according to spatial contact processes or migration. Such propagation of alien tephritids could be responsible for both newly infested cities and recurrences in previously infested cities. We ignored reintroduction of alien tephritids from outside of California because California is a main fruit exporter (CDFA 2017). Besides the local population dynamics and the propagule pressure due to the spatial propagation of alien tephritids from adjacent cities, the probability of infestation in a city also depends on the environmental conditions such as landscape structure and climate each year.

Infested cities can be categorized into experiencing different invasion stages according to the number of detection-years (DY). Some cities have obtained detections over multiple years $(\mathrm{DY}>1)$ while others only experience a single year of detection (DY $=1$; Fig. 1, Appendix S1: Table S1). For simplicity, we thus define the invasion stage (IS) of a city by the number of DYs that it has experienced till a specific year: for instance, a

${ }^{11}$ cdfa.ca.gov city currently at the stage of $\mathrm{IS}_{2}$ has to date detected tephritids in two DYs. The invasion stage of a city, currently at $\mathrm{IS}_{n-1}$, can increase and become $\mathrm{IS}_{n}$ in one of the following years. The transitions from lower to higher invasion stages and from invaded by one species to multispecies in a city capture the invasion dynamics of the city (Fig. 1).

\section{The progression model}

To describe the invasion progression in a cohort of pristine cities, we designed a quantitative progression model based on the HLT of demography (Salomon et al. 2013, Carey and Roach 2019). The progression model is a statistical model that describes the spatial occupation processes of an invasive species. Let $l_{t}$ be the fraction of non-infested cities (thus, $1-l_{t}$ is the fraction of infested cities by invasive species in year $t$ ), which describes the invasion trajectories out of a cohort of pristine cities by a single decrement curve (Fig. 1a, Appendix S1: Table S2):

$$
l_{t}=\frac{N_{t}}{N_{0}}
$$

where $N_{t}$ and $N_{0}$ denote, respectively, the number of non-infested cities in year $t$ and the total number of cities in year zero. For a given IS, we can similarly define $l_{t}($ IS $)$ as the fraction of cities that have not yet reached the IS at year $t$; thus $l_{t}\left(\mathrm{IS}_{n-1}\right) \leq l_{t}\left(\mathrm{IS}_{n}\right)$.

To fit the curves of $l_{t}$ along the year, we chose the logistic model because it provided a better fit to the fraction $l_{t}$ than the Gompertz and Weibull models in the demography of actuarial research (Wilson 1994):

$$
l_{t}^{\prime}=\frac{N_{t}^{\prime}}{N_{0}}=\frac{a_{1}}{1+(t / g)^{\beta}}+a_{2}
$$

with $a_{1}, a_{2}, g$ and $\beta$ model parameters. Note, $N_{t}^{\prime}$ and $l_{t}^{\prime}$ represent predicted number and fraction of non-infested cities from the logistic model in year $t$, respectively. We used the least-squares method for fitting, which minimizes the sum of squares, $\sum_{t=1}^{n}\left|l_{t}^{\prime}-l_{t}\right|^{2},(t=1, \ldots, n$; Rao and Toutenburg 1999). We computed the root mean square error (RMSE), the coefficient of determination $R^{2}$, and the percentage accuracy of the model, $\mathrm{PA}=100 \% \times \frac{1}{n} \sum_{t=1}^{n}\left|\frac{l_{t}-l_{t}^{\prime}}{l_{t}}\right|$, to indicate the goodness of fit. According to the norm of the HLT, we used the predicted fraction of non-infested cities $l_{t}^{\prime}$ in the following analysis. In addition, we also calculated the expected fraction in year 2030 by projecting the fitted logistic model.

We assessed the lag effect between different invasion stages on the invasion dynamics. In particular, the above logistic model allowed us to estimate the year when a given fraction of non-infested cities can be reached,

$$
t_{l^{\prime}}=g \times e^{\left[\frac{1}{\mathrm{~B}} \ln \left(\frac{a_{1}}{t_{t}^{\prime}-a_{2}}-1\right)\right]} .
$$


a) Progression mode

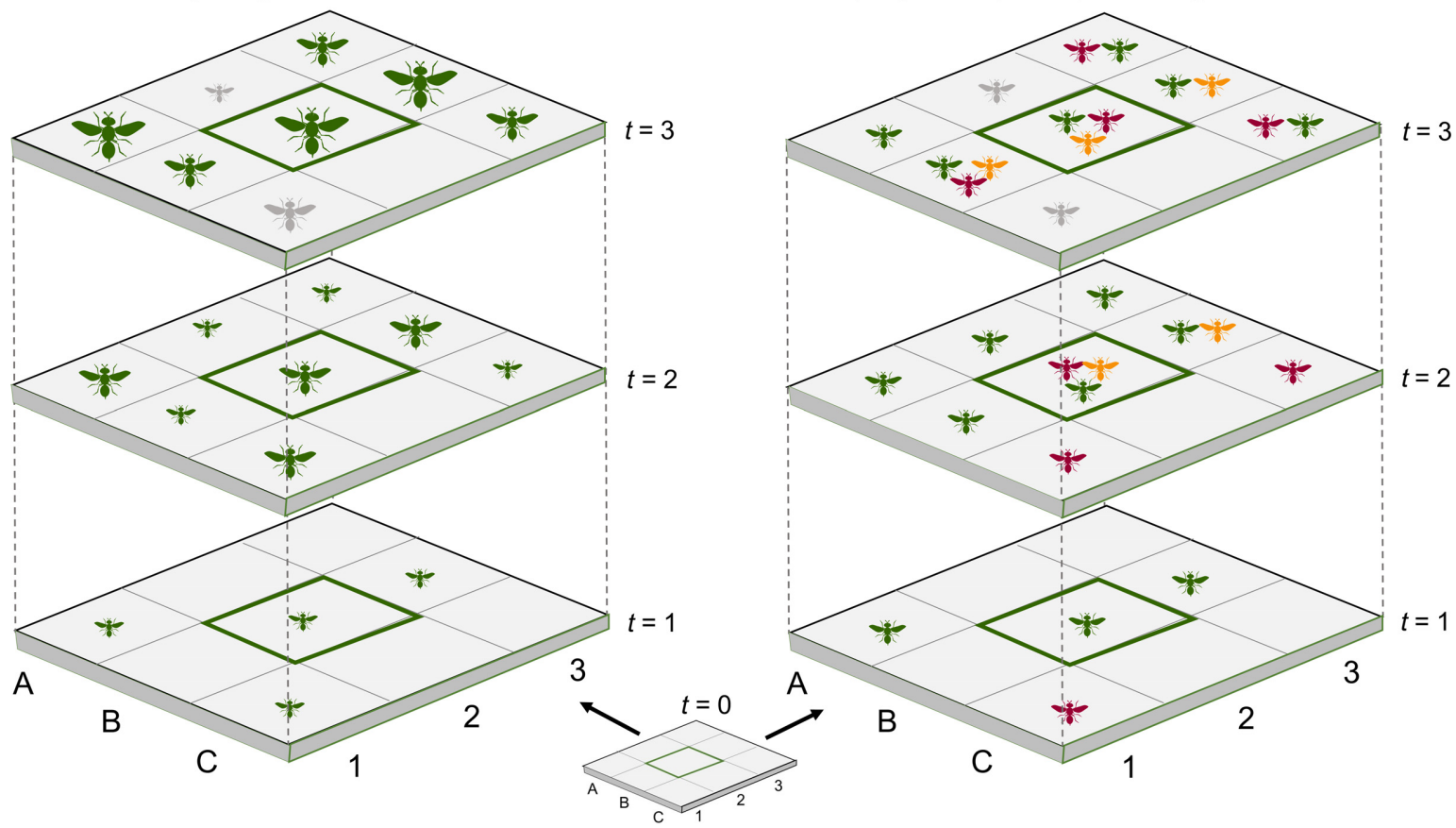

b) Species-specific partitioning model

FIG. 1. Illustrations of basic spatiotemporal concepts underlying each of the two models used in the analysis of fruit fly invasions in California. Cells A1 through C3 depict the spatial boundaries of nine different cities and $t=0$ through $t=3$ denote four different epochs. (a) Progression model. Fly icons indicate detection in one of the nine cities in the three by three grid at time $t$ with the fly's size indicating invasion stage (smallest for IS ${ }_{1}$, first-year detection; mid-sized for $\mathrm{IS}_{2}$, second-year detection; largest for IS 3 , third-year detection). The gray images of flies of different sizes depict the city's stage of invasion based on previous detections but indicate no detections in that cell that year. (b) Partitioning model. Fly icons of different colors indicate different species appearing in one of the nine cities. The patterns over time illustrate the differential species-specific invasion rates and impact on a city's fly invasion processes.

Similarly, for a specific invasion stage IS, we can also fit $l_{t}^{\prime}$ (IS) to the logistic model and estimate the year when a given fraction of cities reaches the specified invasion stage, $t_{l^{\prime}}$ (IS). The lag effect is defined as the time interval $(\Delta t)$ needed for a given fraction of cites $l^{\prime}$ to advance their invasion stage from $\mathrm{IS}_{n-1}$ to $\mathrm{IS}_{n}$ :

$$
\Delta t_{l^{\prime}}=t_{l^{\prime}}\left(\mathrm{IS}_{n-1}\right)-t_{l^{\prime}}\left(\mathrm{IS}_{n}\right)
$$

As each detection in a city was followed by a quarantine treatment, the time interval $(\Delta t)$ from $\mathrm{IS}_{n-1}$ to $\mathrm{IS}_{n}$ transition can also be considered the quarantine interval between consecutive tephritid invasions.

The infestation distribution $\left({ }_{n} d_{t}\right)$ is defined as the fraction of newly infested cities for periods of $n$ years (Appendix S1: Table S2):

$$
{ }_{n} d_{t}=l_{t}-l_{t+n} .
$$

The period infestation rate $\left({ }_{n} q_{t}\right)$ is defined as the growth rate of infested cities in year $t$ :

$$
{ }_{n} q_{t}=\frac{{ }_{n} d_{t}}{l_{t}} .
$$

We normally take $n$ as 1 yr to consider annual rate of invasion infestation, although larger values of $n$ can also be considered. By calculating these metrics $\left(l_{t}, d_{t}\right.$, and $\left.q_{t}\right)$ of the progression model, we can derive accurate parametric form of invasion dynamics and estimate the future invasion patterns of alien species. See Appendix S2for additional details on heuristic models.

\section{The species-specific partitioning model}

A species-specific partitioning model was designed to evaluate species-specific contributions to the multispecies invasions (i.e., transition of cities from pristine to infestation) based on MDLT (Carey 1989). First, the contribution of a species was assessed by removing its records from the data set. In particular, we also used the decremental curves, defined as the decline of noninfested cities along time, to capture the invasion trajectory after removing the records of one species. The fraction of non-infested cities $\left(l_{t}^{-i}=\frac{N_{t}^{-i}}{N_{0}}\right)$ and the infestation distribution $\left({ }_{n} d_{t}^{-i}=l_{t}^{-i}-l_{t+n}^{-i}\right)$ after removing species $i$ were used (Fig. 1b, Appendix S1: Table S3), where variables with the subscript $-i$ were calculated from the data with records of species $i$ removed. Then, invasion trajectories $\left(l^{-i}, d^{-i}\right.$, and $\left.q^{-i}\right)$ with an eliminated species could be compared to the patterns of infested cities with all species included. 
Second, we calculated the potential risk of a specific species in triggering the transition of cities from being pristine to being infested as the following (Fig. 1b, Appendix S1: Table S3):

$$
q_{t}^{i}=1-\frac{l_{t+\infty}^{i}}{l_{t}}=\frac{d_{t}^{i}}{l_{t}} .
$$

Note, ${ }_{\infty} q_{t}^{i}$ means that a city will be eventually infested by species $i$ after year $t$ and therefore represents the potential invasion risk of a city by species $i$.

Third, the relative contribution of species $i$ to infestation was estimated as

$$
{ }_{n} r_{t}^{i}=\frac{{ }_{n} d_{t}^{i}}{{ }_{n} d_{t}}
$$

where ${ }_{n} d_{t}^{i}$ and ${ }_{n} d_{t}$ donate the number of infested cities by species $i$ alone and by all species, respectively, between year $t$ and year $t+n$. This metric ${ }_{n} r_{t}^{i}$ indicates the fraction (competing risk) of infested cities by species $i$.

\section{Statistical analysis}

For demonstration, we separated the 17 tephritids that have been detected in California into four groups based on the numbers of detections: oriental fruit fly, Mediterranean fruit fly, Mexican fruit fly, and others (14 rarely detected species). We produced a Venn diagram (VENNY 2.1) and visualized infested cities invaded

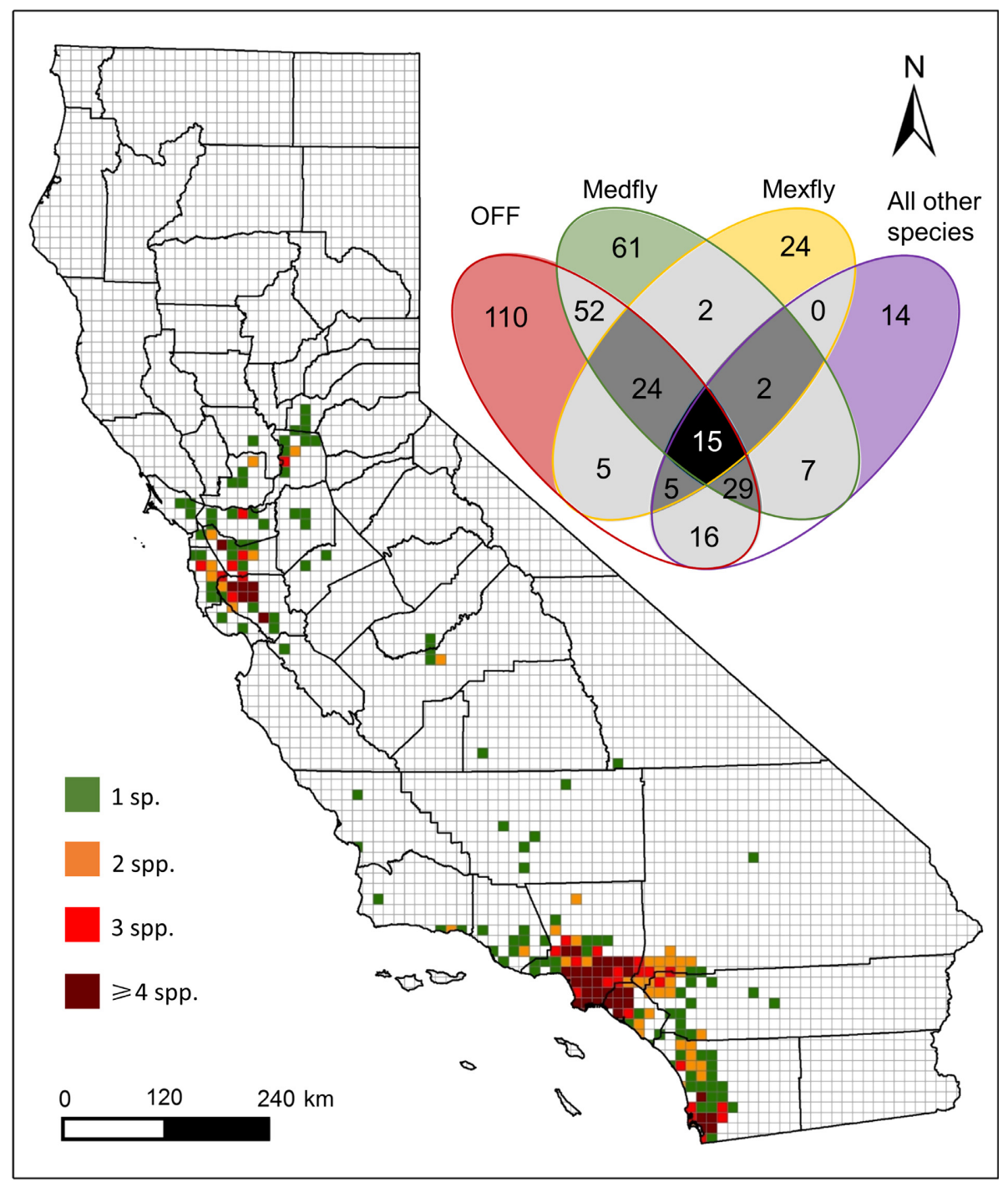

FIG. 2. Species distribution of tephritids and spatial overlap across California cities. Numbers in colored regions indicate how many cities were infested by each of the respective species alone. Number in central area (black) of 100\% overlap indicates the number of cities in which the oriental (OFF), Mediterranean (Medfly), and Mexican fruit flies (Mexfly) plus at least one other tephritid was detected. The numbers in the light gray and dark gray indicated combinations of two and three species, respectively. 
uniquely or jointly by these four groups (Oliveros 2016). The first detection in a city marks the transition from pristine to infested status, while the number of infested cities $\left(N_{t}\right)$ in year $t$ was used to calculate invasion metrics $\left(l_{t}, d_{t}\right.$, and $\left.q_{t}\right)$. The transition from low to high invasion stages (IS) was explored in a similar way to add more aspects of invasion progression. We used the transition probability $P((n+1) \mid n)$ of a city from IS $=n$ to IS $=n+1$, to examine the invasion process of a city (García and Borda-de-Água 2017). Quarantine intervals can be considered the recovery periods of tephritids after launching each emergency eradication program in case of detection. The reduced quarantine intervals indicated failure while increased quarantine intervals made the contrast. Multiple comparisons were employed to examine the differences of quarantine intervals $\left(\mathrm{IS}_{1}-\mathrm{IS}_{2}, \mathrm{IS}_{2}-\right.$ $\left.\mathrm{IS}_{3}, \ldots \mathrm{IS}_{6}-\mathrm{IS}_{7}\right)$ using the glht function in the R package multcomp (Hothorn et al. 2017, R Development Core Team 2017). The quarantine intervals were an effective index to measure the colonization possibility of tephritid population.

\section{Results}

\section{Multispecies invasion patterns}

There are four genera (Anastrepha, Bactrocera, Ceratitis, and Dacus) with 17 tephritid species in California. Of the total of 4,365 detection events, the oriental fruit fly (OFF; first detected in 1960), Mediterranean fruit fly (Medfly; detected in 1975) and Mexican fruit fly (Mexfly; detected in 1954) have been detected, respectively, 1,587, 1,996, and 475 times. The other 14 species accounting for only a total of 307 detection events. The white striped fruit fly (B. albistrigata) was the most recently detected species, only in 2008 (Appendix S1: Table S4, Data S1).

The number of infested cities has been steadily increasing, reaching 366 in 2017. The OFF, Medfly, and Mexfly have invaded 256, 192, and 77 cities, respectively, and exclusively 110,61 , and 24 cites. Additionally, there are 120 cities detected both OFF and Medfly, 49 cities detected both OFF and Mexfly, while Medfly and Mexfly only jointly invaded 43 cities. There are 39 cities infested by all three species (OFF, Medfly, and Mexfly), with 15 cities infested by all four groups (Fig. 2).

\section{Invasion progression}

A total of 366 of the cities experienced infestation $\left(\mathrm{IS}_{1}\right)$, of which 195 cities became infested stage $\mathrm{IS}_{2}$ from the recurrence of tephritids, with 137, 95, 75, and 56 cities then advanced to $\mathrm{IS}_{3}, \mathrm{IS}_{4}, \mathrm{IS}_{5}$, and $\mathrm{IS}_{6}$, respectively (Fig. 3a). The fraction of non-infested cities against the year was accurately described by the logistic model, which was thus used for projecting future invasion tendency (Table 1, Appendix S1: Table S5). The fraction of non-infested cities $\mathrm{IS}_{0}$ decreased continuously, suggesting an ongoing invasion expansion of alien tephritids (Fig. 3a). However, the peak of infestation distribution $\left(d_{t}\right)$ for different IS gradually moved backward in time, with the infestation distribution for $\mathrm{IS}_{1}$ reaching its peak in year 1987 , illustrating a continuously increasing period infestation rate (Fig. 3b, Appendix S1: Fig. S2). The alien tephritids had relatively low infestation distribution during the first three decades (1950-1980) but reached a peak in 1990. In the most recent two decades (2000-2017), the infestation distribution declined again to a low

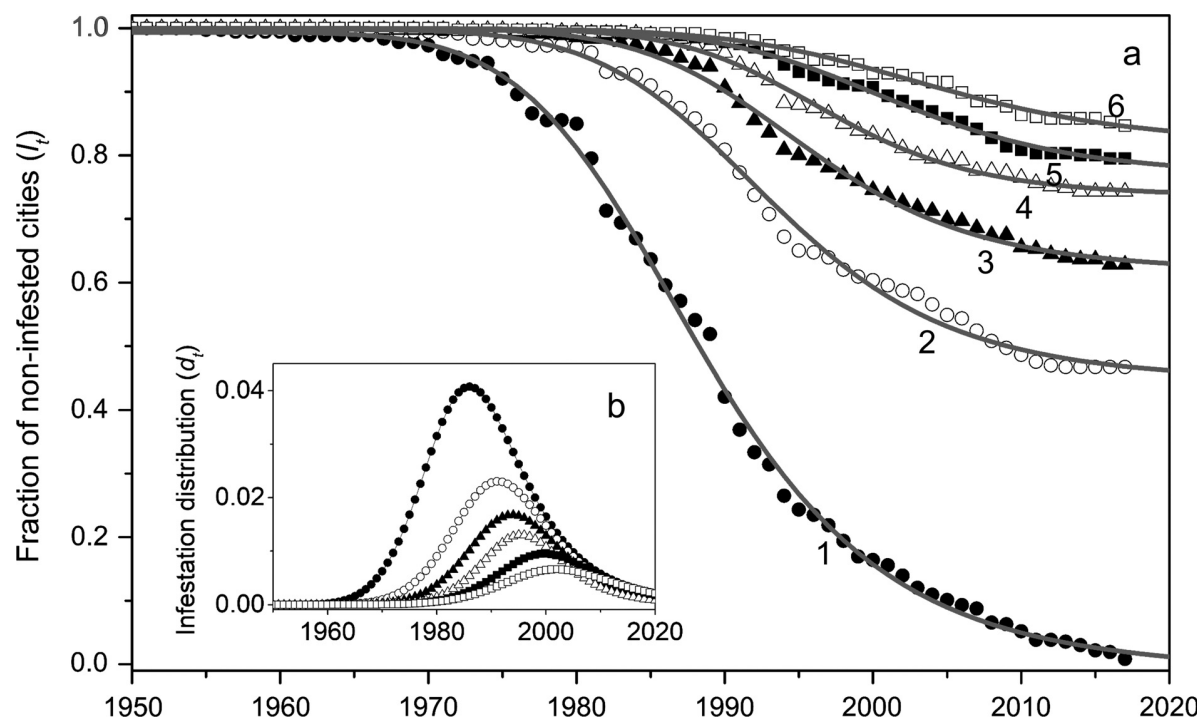

FIG. 3. The invasion processes of progression model: (a) the fraction of non-infested cities and (b) the infestation distribution by alien tephritid invasion in California during 1950 to 2017. The Arabic numerals indicate IS from 1 to 6). 
TABLE 1. The parameters of logistic model for adjusting fraction of non-infested cities and model examination.

\begin{tabular}{lcccccccc}
\hline \hline Categories & $a_{1}$ & \multicolumn{1}{c}{$a_{2}$} & $g$ & $\beta$ & RMSE & PA (\%) & $r$ & \multicolumn{1}{c}{ (\% } \\
\hline IS $_{1}$ & 1.00 & -0.01 & 38.51 & 6.07 & 0.015 & 4.58 & 0.92 & $<0.001$ \\
IS $_{2}$ & 0.55 & 0.44 & 43.41 & 7.08 & 0.013 & 1.48 & 0.91 & $<0.001$ \\
IS $_{3}$ & 0.38 & 0.62 & 45.74 & 7.91 & 0.009 & 0.83 & 0.78 & $<0.001$ \\
IS $_{4}$ & 0.26 & 0.74 & 47.03 & 9.18 & 0.006 & 0.49 & 0.87 & $<0.001$ \\
IS $_{5}$ & 0.23 & 0.77 & 51.65 & 8.39 & 0.005 & 0.42 & 0.93 & $<0.001$ \\
IS $_{6}$ & 0.18 & 0.82 & 54.27 & 7.68 & 0.004 & 0.30 & 0.92 & $<0.001$ \\
\hline
\end{tabular}

Notes: $a 1, a 2, g$ and $\beta$ are model parameters. PA, percentage accuracy; IS, invasion stage.
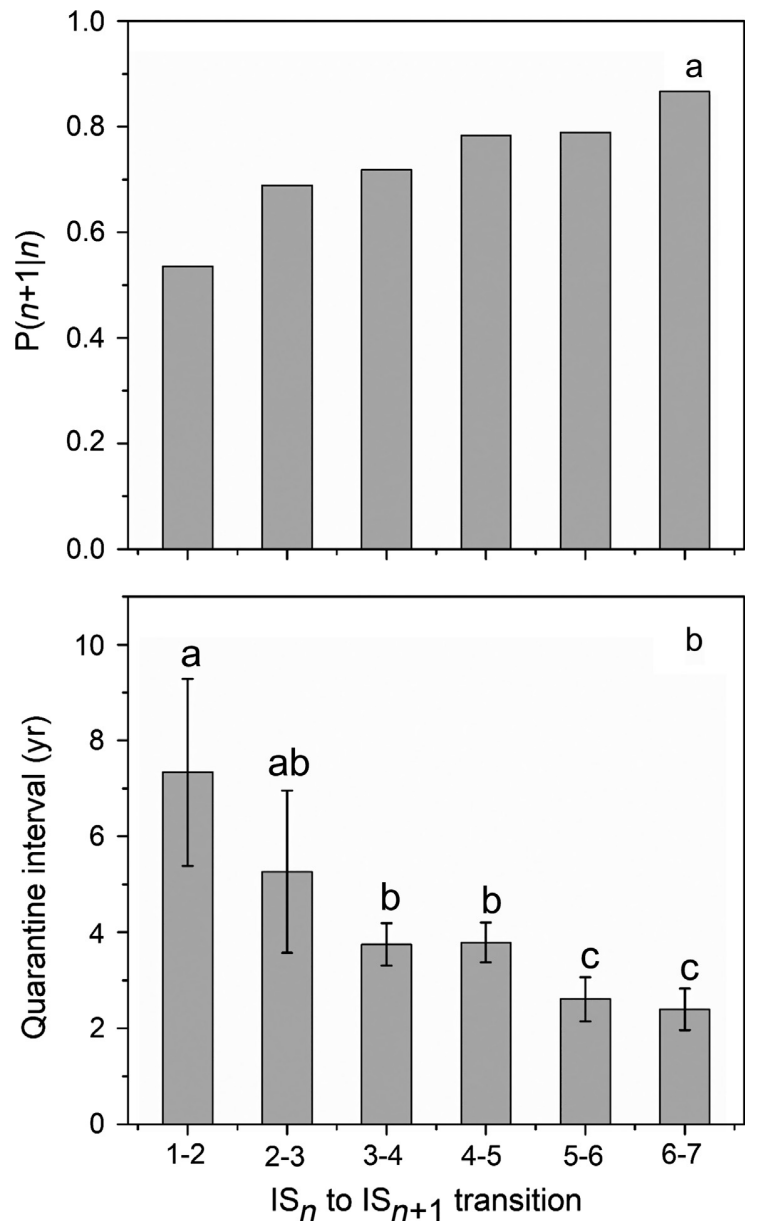

FIG. 4. (a) The invasion stage transition of infested cities and (b) quarantine intervals from $\mathrm{IS}_{n}$ to $\mathrm{IS}_{n+1}$ transition (the same lower-case letters on top of columns indicate no differences while the different letters indicate significant differences at $P \leq 0.05)$.

level, which indicated a relatively slow invasion expansion towards filling up potential suitable habitats.

The DYs of infested cities ranging from 1 to 26 , showing an increasing conditional probability of transition with gradually increasing IS (Fig. 4a). The quarantine interval (lag effect) of infested cities for $\mathrm{IS}_{1}$ to $\mathrm{IS}_{2}$ transition was $7.34 \pm 1.95 \mathrm{yr}$ and declined with increasing IS
$\left(F_{5,605}=86.35, P<0.001\right)$. The quarantine interval for the transition from $\mathrm{IS}_{6}$ to $\mathrm{IS}_{7}$ was merely $2.40 \pm 0.43 \mathrm{yr}$, approaching a biennial outbreak of alien tephritids in cities with advanced invasion stages (Fig. 4b). The constantly reduced quarantine intervals indicated that eradication program did not change the invasion progression of alien tephritids.

\section{Invasion partitioning}

The invasion process of a single tephritid species can be captured by a single decrement curve (Fig. 5a). The OFF and Mexfly had very similar single decrement curves while Medfly had a sharp decreasing period during the decades from 1990 to 2000 . The results of the species-elimination revealed that the number of infested cities was reduced from 366 to 258 when the OFF was removed; removal of the Mexfly only caused a small change in patterns of infested cities, reducing the number from 366 to 346 (Fig. 5b). Removing both OFF and Medfly led to a sharp decrease in the number of infested cities from 366 to 144 while infested cities decreased to 93 after three species eliminations (Fig. 5c, d).

In total, the OFF, Medfly, and Mexfly accounted for $49.5 \%, 31.1 \%$, and $9.3 \%$ infested cities while the other 14 species combined accounted for $10.1 \%$ of infested cities (Fig. 6a). The OFF peaked in 2000 and 2010, indicating a high percentage of infested cities $\left({ }_{n} r_{t}^{i}\right)$ among four groups while the Medfly had an increasing proportion in recent years (Fig. 6b). Therefore, the OFF has the most invasion potential than other groups, indicating it the most dominant invasive tephritids in California.

\section{Discussion}

\section{Methodological context}

Our life-table invasion models address spatiotemporal processes of invasive alien species, which have received substantial attention from ecologists (Roques et al. 2016, Sullivan et al. 2017). There are at least two reasons why the life-table models can have such a conceptual and methodological reach and, therefore can be brought to bear on questions in invasion science (Carey and Roach 2019). First, we used the invasion transition of cities from a pristine stage to an IS in the context of 
a) None eliminated

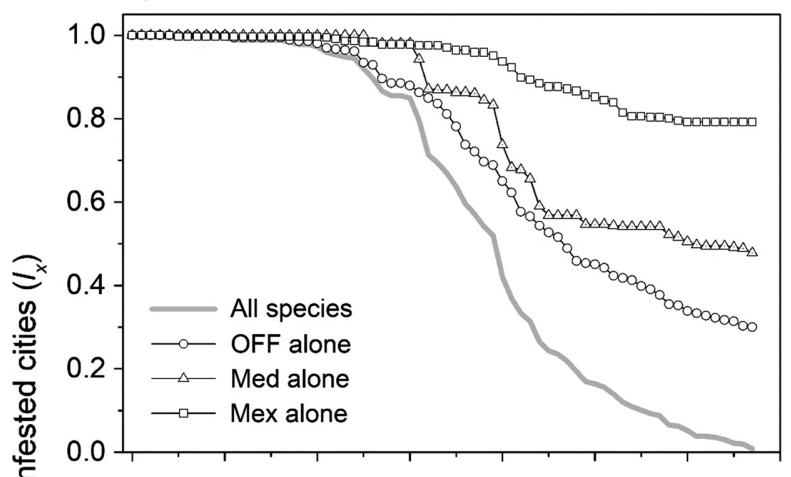

c) Two-species elimination

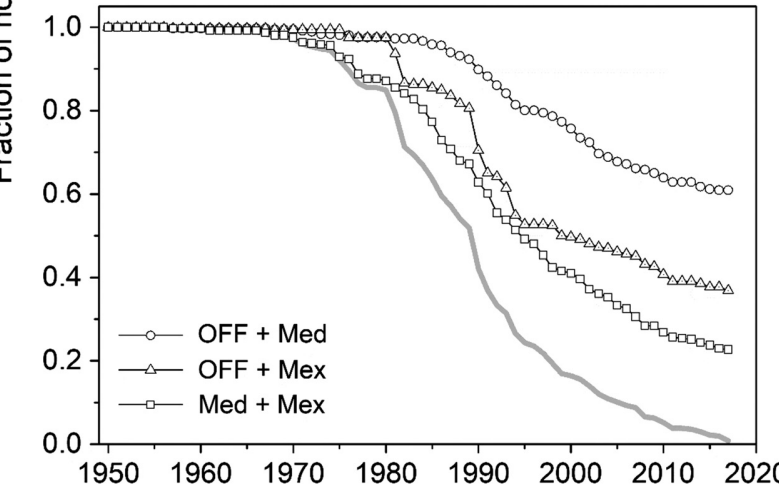

b) One-species elimination

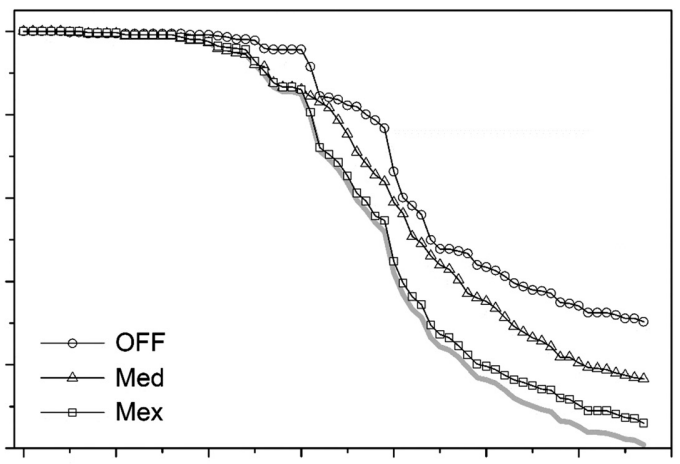

d) Three-species elimination

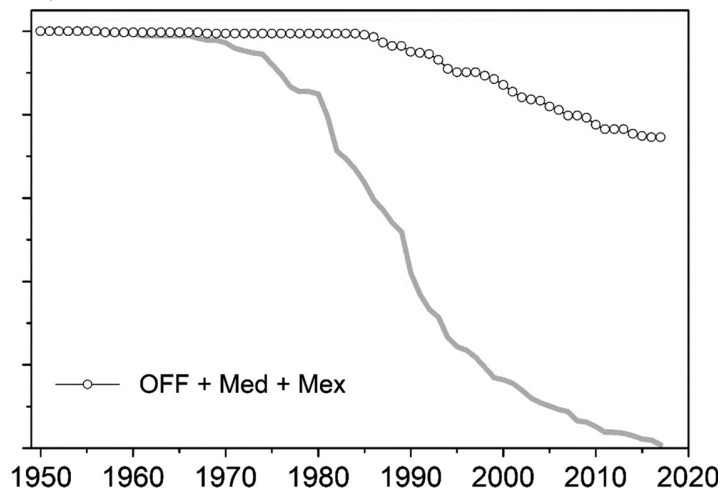

FIG. 5. The fraction of non-infested cities from $t$ to $t+1$ when species $i$ is eliminated: (a) all species (no elimination) and three dominant species alone, (b) one-species elimination, (c) two-species elimination, (d) three-species elimination. OFF, Oriental fruit fly, Med, Mediterranean fruit fly, and Mex, Mexican fruit fly.

either recurrence or infestation by multispecies based on discrete entity in a variety of contexts. So, the spatial expansion of alien tephritids could be resolved by analyzing $\mathrm{IS}_{0}$ to $\mathrm{IS}_{1}$ transitions while incessant recurrences indicate elevated invasion adaptation after repeated eradications. Therefore, invasion stages (IS) can provide useful information to policy making that any eradication programs should be adaptive to the detection history of a city or region rather than be fixed and static (Yamamura et al. 2016). Second, our models generate complementary sets of metrics that could reveal different types of information about the invasion processes (e.g., infestation distribution $\left[d_{t}\right]$ and period infestation rate $\left[q_{t}\right]$ ), providing a quantitative platform to compare invasion trajectories of multiple species (Land et al. 2005).

\section{Invasion progression model}

Our invasion progression model highlights the necessity to grade IS when exploring invasion dynamics. Invasion progression has different IS ranged from noninfestation to many detection-years. A city that has not been invaded is at a much different IS than one that has previously experienced even a single year of detection, not mentioning those with multiple years of detections (Lambdon and Hulme 2006). This highlights the importance of past invasion dynamics and the potential inertia of invasion recurrence. The concept of gradations of IS allows cities to be classified both qualitatively and quantitatively from low to high IS based on the number of detection-years. Invasion risk analyses using established species distribution models should thus consider the role of IS in future development as the probabilities of initial and recurrent invasions are clearly not solely dependent on environmental suitability (Schliep et al. 2018).

The invasion progression model could not only estimate invasion expansion, but also predict transition between different IS. This information allows us to embed each city's current invasion risk in the context of its long-term invasion history and future trend (CDFA 2018). Our model revealed the quarantine intervals of cities that have experienced more than $1 \mathrm{yr}$ of detection are decreasing with increasing number of detection-years (Fig. 3); this indicates that the tephritids have firmly established in California (Papadopoulos et al. 2013) and that new detections are the results of failed suppression of an established, environmentally adapted resident population (Sutherland et al. 2018). Frequent detections of tephritids mean these populations have become adapted 

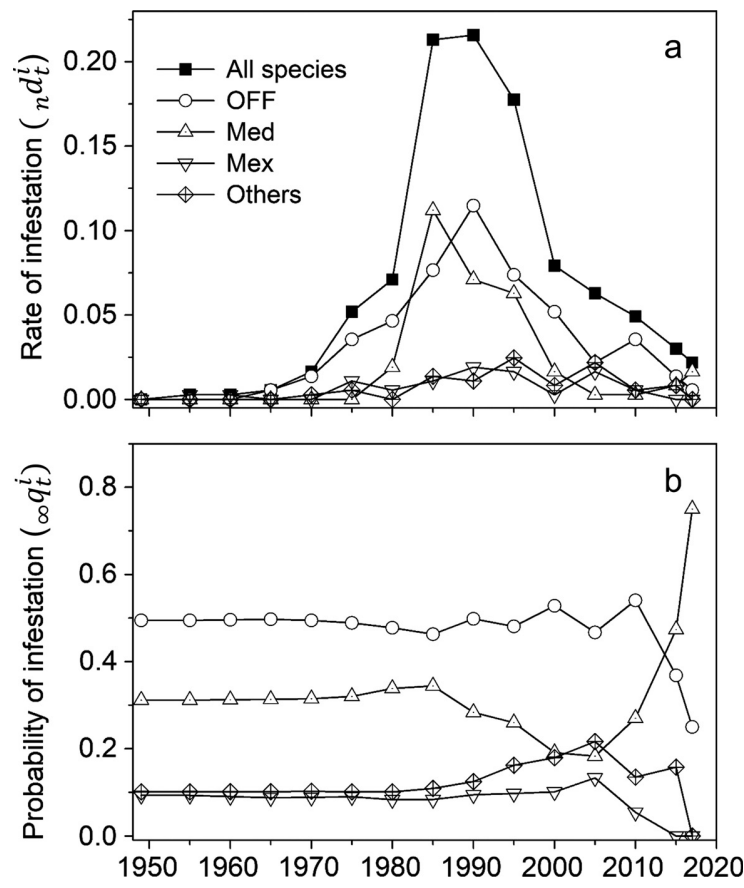

FIG. 6. Contribution of each alien tephritid species on infested cities based on the species-specific partitioning model. (a) Infestation distribution of each species and (b) probability of eventual infestation due to species-specific invasions.

to California (Carey et al. 2017), enabling tephritids to recover their population after each emergency eradication program. Such decreasing quarantine interval between detections indicates the rising risk of additional recurrent invasions; it also follows that eradication programs were not truly extirpating these pests from California.

Our progression model can also be used to examine other regional invasions with detailed data available on both spatial distributions and temporal dynamics (e.g., gypsy moth [Lymantria dispar] and Colorado potato beetle [Leptinotarsa decemlineata]) and explore the role of recurrent outbreaks in ongoing invasion expansion. It further provides a quantitative framework for formulating invasion debt and illustrating spatial processes (Rigot et al. 2014, Roques et al. 2016) and thus provides support for proactive management of biological invasions.

\section{Species-specific invasion partitioning}

Although multispecies invasions have been widely reported in many places (Follett and Zee 2011, Papadopoulos et al. 2013), our method allows us for the first time to quantitatively partition the entangled multispecies invasions into species-specific contributions. Evidently, multispecies invasions could complicate invasion and/or successional trajectories of particular species due to invasional meltdown or synergistic interactions (Rivett et al. 2018). As species-specific traits and their environmental responses create contextual interspecific interactions and thus aggravate the complexity of multispecies invasions, distinguishing good and bad invaders could help refine trait-based prediction of alien species (Pearson et al. 2016). Our partitioning model shed new light in comparative invasion ecology on what makes a good invader (Williamson and Fitter 1996). The invasion partitioning model revealed large discrepancies between species in their invasion capabilities, and such quantitatively measured discrepancies in invasion capabilities could reflect both trait differences and other related invasion factors and should be used as a key dependent variable in future invasion ecology to replace the current discrete status along introduction-naturalization-invasion continuum (Richardson and Pyšek 2008). In addition, to explore multispecies invasions especially in the setting of an ecological network (Hui and Richardson 2019), it is rather challenging to work out pairwise interaction strengths, while the partitioning model could tentatively provide indirect indicators of interaction strengths based on coinvasion patterns.

Our result identified Medfly being accounted for most infestations after 2010 as compared to other three groups (Fig. 6b); this enables more effective allocation of eradication efforts based on species-specific strategies. It could also potentially provide ecologists with information about the invasion trajectory and distributions of each species with the species-elimination method (Fig. 5, Appendix S1: Figs. S3-S6).

\section{Conclusions}

Our models provided evidence for illustrating both spatial expansion and species-specific partitioning of multispecies invasions, which had many advantages due to maneuverability and applicability. Our models with three parameters $\left(l_{t}, d_{t}\right.$, and $\left.q_{t}\right)$ provide a clear, intuitive, and transparent framework for quantitatively characterizing general trends in progression and invasion stage transitions. Our models also encourage comparisons of invasion trajectories among different stages or between different species. The progression model reveals invasion stage transitions from less-infested to much-infested cities, while the partitioning model could distinguish species-specific invasions of multispecies.

Taken together, by solving both progression and species-specific partitioning simultaneously, our models clearly illuminated multispecies invasions that are often assessed based on descriptive or biased expansion rate due to mixed species, habitat and single spatial occupation specificity (Rivett et al. 2018). Consequently, our models have potential for quantitative estimation of spatiotemporal invasions, which could also be applied to illustrate interspecific interactions between species. We believe that one of the most important overarching contributions of our models is not only the introduction of novel applications of demographic models to invasion biology, but also the conceptual 
and analytical linkage these models provide to actual populations of alien species. Indeed, this approach may lay the groundwork for the development of a hierarchical demography of invasions, which is to say populations within populations.

\section{ACKNOWLEDGMENTS}

We thank the California Department of Food and Agriculture for providing us with tephritid capture data. We also thank Christian Nansen and Sarah Silverman at the Department of Entomology, UC Davis for comments on invasion analysis. Z. Zhao and J. R. Carey designed the experiments. Z. Zhao arranged the data, constructed the models, conducted the statistical analysis, and wrote the first draft. All authors reviewed, revised, approved the final version and declared that they have no competing interests. Z. Zhao was partially supported by the National Key R \& D Program of China (No. 2017YFD0200400), the National Science Foundation of China (No. 31770453; 31770470), and Key R\&D Projects of Ningxia Hui Autonomous Region (No. 2017BY080).

\section{Literature Cited}

Amin, M. B., F. L. Greene, S. B. Edge, C. C. Compton, J. E. Gershenwald, R. K. Brookland, L. Meyer, D. M. Gress, D. R. Byrd, and D. P. Winchester. 2017. The eighth edition AJCC cancer staging manual: continuing to build a bridge from a population-based to a more "personalized" approach to cancer staging. CA: A Cancer Journal for Clinicians 67:93-99.

Bellows, T. S., R. G. Vandriesche, and J. S. Elkinton. 1992. Lifetable construction and analysis in the evaluation of natural enemies. Annual Review of Entomology 37:587-614.

Bertelsmeier, C., and L. Keller. 2018. Bridgehead effects and role of adaptive evolution in invasive populations. Trends in Ecology and Evolution 33:527-534.

Carey, J. R. 1989. The multiple decrement life table: a unifying framework for cause-of-death analysis in ecology. Oecologia 78:131-137.

Carey, J. R. 2001. Insect biodemography. Annual Review of Entomology 46:79-110.

Carey, J. R., and D. Roach. 2019. Biodemography: introduction to concepts and methods. Princeton University Press, Princeton, New Jersey, USA.

Carey, J. R., N. Papadopoulos, and R. E. Plant. 2017. The 30year debate on a multi-billion-dollar threat: Tephritid fruit fly establishment in California. American Entomologist 63:100 113.

Cassey, P., S. Delean, J. L. Lockwood, J. S. Sadowski, and T. M Blackburn. 2018. Dissecting the null model for biological invasions: a meta-analysis of the propagule pressure effect. PLoS Biology 16:e2005987.

CDFA. 2017. California Agricultural Exports 2016-2017. California Agricultural Statistics Review 2017-2018. CDFA, Sacramento, California, USA.

CDFA. 2018. California Department of Food and Agriculture Quarantine Manual, Section 3423. Oriental fruit fly interior quarantine. CDFA, Sacramento, California, USA.

Chi, H. 1988. Life-table analysis incorporating both sexes and variable development rates among individuals. Environmental Entomology 17:26-34.

Coutsoudis, A., K. Pillay, E. Spooner, L. Kuhn, and H. M. Coovadia. 1999. Influence of infant-feeding patterns on early mother-to-child transmission of HIV-1 in Durban,
South Africa: a prospective cohort study. Lancet 354:471476.

Dantonio, C. M., and P. M. Vitousek. 1992. Biological invasions by exotic grasses, the grass fire cycle, and global change. Annual Review of Ecology and Systematics 23:63-87.

Duan, J. J., K. J. Abell, L. S. Bauer, J. Gould, and R. Van Driesche. 2014. Natural enemies implicated in the regulation of an invasive pest: a life table analysis of the population dynamics of the emerald ash borer. Agricultural and Forest Entomology 16:406-416.

Epanchin-Niell, R. S., and A. M. Liebhold. 2015. Benefits of invasion prevention: effect of time lags, spread rates, and damage persistence. Ecological Economics 116:146-153.

Ergon, T., Ø. Borgan, C. R. Nater, and Y. Vindenes. 2018. The utility of mortality hazard rates in population analyses. Methods in Ecology and Evolution 00:1-11.

Follett, P. A., and F. T. Zee. 2011. Host status of Vaccinium reticulatum (Ericaceae) to invasive tephritid fruit flies in Hawaii. Journal of Economic Entomology 104:571-573.

García, C., and L. Borda-de-Água. 2017. Extended dispersal kernels in a changing world: insights from statistics of extremes. Journal of Ecology 105:63-74.

Graunt, J. 1662. Natural and political observations mentioned in a following index, and made upon the bills of mortality. Journal of the Institute of Actuaries 90: 1-61.

Hothorn, T., F. Bretz, P. Westfall, R. M. Heiberger, A. Schuetzen-Meister, and S. Scheibe. 2017. Package multcomp. Simultaneous inference in general parametric models. Version 1.4-8. https://cran.r-project.org/web/packages/multcomp/index. html

Hui, C., and D. M. Richardson. 2017. Invasion dynamics. Oxford University Press, Oxford, UK.

Hui, C., and D. M. Richardson. 2019. How to invade an ecological network. Trends in Ecology and Evolution 34:121-131.

Kearney, M., and W. Porter. 2009. Mechanistic niche modelling: combining physiological and spatial data to predict species' ranges. Ecology Letters 12:334-350.

Koons, D. N., M. Gamelon, J. M. Gaillard, L. M. Aubry, R. F. Rockwell, F. Klein, R. Choquet, and O. Gimenez. 2014. Methods for studying cause-specific senescence in the wild. Methods in Ecology and Evolution 5:924-933.

Kriticos, D. J., and A. Leriche. 2010. The effects of climate data precision on fitting and projecting species niche models. Ecography 33:115-127.

Lamb, V. L., and J. S. Siegel. 2004. Health demography. Pages 341-370 in J. S. Siegel, and D. A. Swanson, editors. The methods and materials of demography. Elsevier Academic Press, Amsterdam, Netherlands.

Lambdon, P. W., and P. E. Hulme. 2006. Predicting the invasion success of Mediterranean alien plants from their introduction characteristics. Ecography 29:853-865.

Land, K. C., Y. Yang, and Z. Yi. 2005. Mathematical demography. Pages 659-717 in D. Poston and M. Micklin, editors. Handbook of population. Springer, New York, New York, USA.

Lloyd-Jones, D. M., M. G. Larson, A. Beiser, and D. Levy. 1999. Lifetime risk of developing coronary heart disease. Lancet 353:89-92.

Manrakhan, A., J. H. Venter, and V. Hattingh. 2015. The progressive invasion of Bactrocera dorsalis (Diptera: Tephritidae) in South Africa. Biological Invasions 17:2803-2809.

McGeoch, M. A., P. Genovesi, P. J. Bellingham, M. J. Costello, C. McGrannachan, and A. Sheppard. 2016. Prioritizing species, pathways, and sites to achieve conservation targets for biological invasion. Biological Invasions 18:299-314. 
Oliveros, J. C. 2016. Venny. An interactive tool for comparing lists with Venn's diagrams. 2007-2015. BioinfoGP, CNBCSIC. http://bioinfogp.cnb.csic.es/tools/venny/

Papadopoulos, N. T., R. E. Plant, and J. R. Carey. 2013. From trickle to flood: the large-scale, cryptic invasion of California by tropical fruit flies. Proceedings of the Royal Society B 280:20131466.

Pearson, D. E., Y. K. Ortega, O. Eren, and J. L. Hierro. 2016. Quantifying "apparent" impact and distinguishing impact from invasiveness in multispecies plant invasions. Ecological Applications 26:162-173.

Peterson, R. K. D., R. S. Davis, L. G. Higley, and O. A. Fernandes. 2009. Mortality risk in insects. Environmental Entomology 38:2-10.

Preston, S. H., P. Heuveline, and M. Guillot. 2001. Demography: measuring and modeling population processes. Blackwell Publishers, Malden, Massachusetts, USA.

R Development Core Team. 2017. R: A language and environment for statistical computing. R Foundation for Statistical Computing, Vienna, Austria.

Rao, R. C., and H. Toutenburg. 1999. Linear models : least squares and alternatives. Springer, New York, New York, USA.

Richardson, D. M., and P. Pyšek. 2008. Fifty years of invasion ecology - the legacy of Charles Elton. Diversity and Distributions 14:161-168.

Rigot, T., I. van Halder, and H. Jactel. 2014. Landscape diversity slows the spread of an invasive forest pest species. Ecography 37:648-658.

Rivett, D. W., M. L. Jones, J. Ramoneda, S. B. Mombrikotb, E. Ransome, and T. Bell. 2018. Elevated success of multispecies bacterial invasions impacts community composition during ecological succession. Ecology Letters 21:516-524.

Roques, A., M. A. Auger-Rozenberg, T. M. Blackburn, J. Garnas, P. Pysek, W. Rabitsch, D. M. Richardson, M. J. Wingfield, A. M. Liebhold, and R. P. Duncan. 2016. Temporal and interspecific variation in rates of spread for insect species invading Europe during the last 200 years. Biological Invasions 18:907-920.

Salomon, J. A., H. Wang, and M. K. Freeman. 2013. Healthy life expectancy for 187 countries, 1990-2010: a systematic analysis for the Global Burden of Disease Study 2010. Lancet 381:628-628.

Schliep, E. M., N. K. Lany, P. L. Zarnetske, R. N. Schaeffer, C. M. Orians, D. A. Orwig, and E. L. Preisser. 2018. Joint species distribution modelling for spatio-temporal occurrence and ordinal abundance data. Global Ecology and Biogeography $27: 142-155$.

Simberloff, D. 2006. Invasional meltdown 6 years later: important phenomenon, unfortunate metaphor, or both? Ecology Letters 9:912-919.

Steiner, L. F. 1952. Methyl eugenol as an attractant for oriental fruit fly. Journal of Economic Entomology 45:241-248.

Sullivan, L. L., B. T. Li, T. E. X. Miller, M. G. Neubert, and A. K. Shaw. 2017. Density dependence in demography and dispersal generates fluctuating invasion speeds. Proceedings of the National Academy of Sciences USA 114:5053-5058.

Sutherland, W. J., et al. 2018. A 2018 Horizon scan of emerging issues for global conservation and biological diversity. Trends in Ecology and Evolution 33:47-58.

Thomas, S. M., G. S. Simmons, and M. P. Daugherty. 2017. Spatiotemporal distribution of an invasive insect in an urban landscape: introduction, establishment and impact. Landscape Ecology 32:2041-2057.

Vicente, J. R., D. Alagador, C. Guerra, J. M. Alonso, C. Kueffer, A. S. Vaz, R. F. Fernandes, J. A. Cabral, M. B. Araujo, and J. P. Honrado. 2016. Cost-effective monitoring of biological invasions under global change: a model-based framework. Journal of Applied Ecology 53:1317-1329.

WHO. 2001. International classification of functioning, disability and health. World Health Organization, Geneva, Switzerland.

Williamson, M. H., and A. Fitter. 1996. The varying success of invaders. Ecology 77:1661-1666.

Wilson, D. L. 1994. The analysis of survival (mortality) datafitting Gompertz, Weibull, and logistic functions. Mechanisms of Ageing and Development 74:15-33.

Yamamura, K., H. Katsumata, J. Yoshioka, T. Yuda, and K. Kasugai. 2016. Sampling inspection to prevent the invasion of alien pests: statistical theory of import plant quarantine systems in Japan. Population Ecology 58:6380.

Zeng, Y., G. V. P. Reddy, Z. Li, Y. Qin, Y. Wang, X. Pan, F. Jiang, F. Gao, and Z. Zhao. 2019. Global distribution and invasion pattern of oriental fruit fly, Bactrocera dorsalis (Diptera: Tephritidae). Journal of Applied Entomology 143:1-12.

\section{SUPPORTING INFORMATION}

Additional supporting information may be found in the online version of this article at http://onlinelibrary.wiley.com/doi/ 10.1002/ecy.2682/suppinfo

\section{Data Availability}

